\title{
Microalbuminuria and Glomerular Filtration Rate in Paediatric Visceral Leishmaniasis
}

\author{
Neena Verma, ${ }^{1}$ Chandra Shekhar Lal, ${ }^{1,2}$ Vidyanand Rabidas, ${ }^{1}$ Krishna Pandey, ${ }^{1}$ \\ Dharmendra Singh, ${ }^{1}$ Sanjay Kumar, ${ }^{1}$ Rakesh Bihari Verma, ${ }^{1}$ and Pradeep Das ${ }^{1}$ \\ ${ }^{1}$ Rajendra Memorial Research Institute of Medical Sciences (Indian Council of Medical Research), Agam Kuan, Patna 800 007, India \\ ${ }^{2}$ Laboratory of Clinical Biochemistry, Rajendra Memorial Research Institute of Medical Sciences (Indian Council of Medical Research), \\ Agam Kuan, Patna 800 007, India
}

Correspondence should be addressed to Chandra Shekhar Lal; drcslal@sify.com

Received 29 April 2013; Accepted 10 June 2013

Academic Editor: Subash Babu

Copyright (c) 2013 Neena Verma et al. This is an open access article distributed under the Creative Commons Attribution License, which permits unrestricted use, distribution, and reproduction in any medium, provided the original work is properly cited.

\begin{abstract}
Visceral leishmaniasis, caused by Leishmania donovani, is a serious form of leishmaniasis and fatal if untreated. Nearly half of the VL cases are children. There are very few studies of renal function in pediatric visceral leishmaniasis. The aim of this study was to evaluate renal dysfunction by studying glomerular filtration rate (GFR), microalbuminuria, and microscopic examination of urine. Laboratory analysis was performed on blood and urine samples of 40 parasitologically confirmed pediatric VL cases. Laboratory data of urine examination showed albuminuria in $10 \%(4 / 40)$, white blood cells in $20 \%(8 / 40)$, hematuria in $10 \%$ (4/40), microalbuminuria in $37.5 \%$ (15/40), and decreased GFR in $27.5 \%$ (11/40). Renal involvement was manifested in most of the pediatric VL cases. These findings may help clinicians in decision making for safe and suitable antileishmanial treatment particularly in childhood VL.
\end{abstract}

\section{Introduction}

The leishmaniases, an infectious disease endemic in tropical, Asian, and southern European countries, is caused by obligate intramacrophage protozoa and is transmitted through the bite of infected female sandflies. The disease phenotypes include visceral leishmaniasis (VL), post-kala-azar dermal leishmaniasis (PKDL), cutaneous leishmaniasis (CL), and mucosal leishmaniasis (ML). Visceral leishmaniasis (VL), caused by Leishmania donovani, is the most serious, mainly affects children, and is fatal if untreated. Nearly half of the VL cases occur in children (childhood or paediatric VL) [1]. The disease is prevalent over large areas of Bihar, India. VL is a febrile illness that is characterized by weight loss, pancytopenia, hepatosplenomegaly, and lymphadenopathy and can be complicated by acute renal damage [2-4]. Even though kala-azar nephropathy is still poorly understood, kidney dysfunction in this disease has been reported in several studies. Immune complex deposition, $\mathrm{T}$ cells, and adhesion molecules activation have been shown to be important mechanisms of injury in glomerulonephritis occurring in visceral leishmaniasis [5-9].

One of the first human studies about renal involvement in kala-azar was a cohort study of 50 patients with visceral leishmaniasis [10]. Proteinuria and/or microscopic haematuria or pyuria was observed in $51 \%$ of patients. Urinary protein excretion was elevated in $57 \%$ of patients, and in all of them, this was $<1 \mathrm{~g} / 24 \mathrm{~h}$. Renal function was evaluated in 11 patients with visceral leishmaniasis, and results stated that 5 patients presented macroscopic haematuria and 1 developed acute nephritic syndrome [11], while urine examination showed proteinuria in 10 patients and haematuria and leukocyturia in 7 and 6 cases, respectively. In a cross-sectional study of 50 patients with chronic visceral leishmaniasis, a decreased glomerular filtration rate (GFR) in $28 \%$ of the cases is reported [12]. More recently, the presence of increased albumin excretion in $44 \%$ of patients [13] was 
observed. The glomerular damage may manifest as mesangioproliferative or membranoproliferative glomerulonephritis due to depositions of immune complexes in the glomeruli. Moreover, tubulointerstitial nephritis may occur and consists of tubular degeneration and inflammatory infiltration with renal impairment $[2,5,14-16]$. Acute kidney injury had been observed in children with visceral leishmaniasis [17]. Paediatric VL patients are found more in numbers in Bihar, India, and studies examining nephrotoxicity in children are scarce.

Conventional amphotericin B (AmB) is the treatment of choice for antileishmanial treatment in Bihar, India. This drug possesses high antileishmanial efficacy, but it is associated with a high risk of renal toxicity in addition to other side effects (rigor, fever, malaise, anorexia, thrombophlebitis, and bone marrow suppression) [18]. In addition to the poor selectivity of amphotericin B between human cholesterol and fungal ergosterol, other mechanisms seem to be involved in the pathogenesis of renal toxicity of the drug [19]. In India, liposomal amphotericin B showed lower rates of toxicity than conventional amphotericin B or amphotericin B lipid complex [20-23]. Hence, keeping in mind the selectivity of amphotericin B with human cholesterol and hypocholesterolemia in paediatric visceral leishmaniasis [24], we examined pediatric VL patients for glomerular involvement before start of AmB treatment. This will be the first ever information about renal injury in pediatric VL patients from this part of India.

Kidney disease is mostly silent, common and harmful but treatable. The only reason for requesting creatinine is to assess kidney function. Routine reporting of estimated glomerular filtration rate (GFR) alongside creatinine has proved to be a useful tool for clinicians in the detection and management of kidney injury. On the other hand, microalbuminuria (MA) is considered as an early marker of glomerulonephritis and is defined as a persistent elevation of albumin in urine of 30$300 \mathrm{mg} /$ day.

The aim of our study is to determine the prevalence of renal injury in paediatric VL patients of Bihar, India, who are mostly being treated in the endemic areas by $\mathrm{AmB}$ and evaluate it according to GFR and microalbuminuria for the purpose of early detection with regards to renal injury.

\section{Materials and Methods}

2.1. Patients and Samples. The observational study included pediatric VL patients aged between 2 and 14 yrs that admitted to the indoor ward of Rajendra Memorial Research Institute of Medical Sciences (Indian Council of Medical Research) Patna, India, for antileishmanial treatment by Amphotericin B deoxycholate. Altogether, 40 subjects were selected for this study after obtaining informed consent from each patient's next of kin. The serum, fresh urine and 24-hour urine, samples were collected from 40 confirmed paediatric VL patients microscopically positive for Leishmania donovani parasite in splenic/bone marrow aspirate before onset of antileishmanial treatment. Urine analysis, serum creatinine, and urea were measured in all patients. The samples were kept in refrigerator $\left(2-8^{\circ} \mathrm{C}\right)$ until assayed.

2.2. Urine Analysis. Urine analysis was done on a fresh morning sample of urine within one hour of collection in a sterile dry container. Physical examination for colour, appearance, and any sediment formation was done. Urine was mixed properly and tested by URiSCAN urine strips (YD Diagnostics, Republic of Korea) for chemical examination, that is, bilirubin, urobilinogen, ketone, protein (albumin), nitrite, glucose, $\mathrm{pH}$, specific gravity, leukocytes, and blood as per the procedure mentioned in the leaflet insert.

2.3. Microscopic Examination of Urine Sample. About $5 \mathrm{~mL}$ of properly mixed urine sample was centrifuged in a centrifuge tube for $5 \mathrm{~min}$ at 2,500 rpm. Supernatant was poured off, and one drop of shaked deposit was placed on a glass slide for microscopic examination under low and high power objectives (10-15 fields) for the presence of white blood cells (WBCs), erythrocytes (RBCs), casts (granular, hyaline, cellular, etc.), epithelial cells, crystals, and so forth per high power field.

2.4. Estimated Glomerular Filtration Rate (eGFR) Measurement. eGFR was measured in children using Schwartz formula. This employs the serum creatinine $(\mathrm{mg} / \mathrm{dL})$, the child's height $(\mathrm{cm})$, and a constant to estimate the glomerular filtration rate:

$$
\mathrm{eGFR}=\frac{k \times \text { Height }}{\text { Serum Creatinine }},
$$

where $k$ is a constant that depends on muscle mass. For infants and children of age 1 to 12 years, $k=0.55$.

2.5. Microalbuminuria Measurement. A 24-hour urine sample was collected from the study subjects in clean plastic containers with boric acid as preservative. The urine samples were kept in the refrigerator till analysis. A commercial turbidometric kit (Tulip Diagnostic, Goa, India) was used for the measurement of MA. The company's instructions were strictly adhered to. One $\mathrm{mL}$ of reagent buffer (polyethylene glycol, Tris/HCL buffer, and sodium chloride) was pipette into seven different test tubes (6 tubes for standard, 1 test tube for sample), and $0.1 \mathrm{~mL}$ of standards (human serum albumin) with different concentrations (5, 10, 20, 50, 100, and $200 \mu \mathrm{g}$ ) and $0.1 \mathrm{~mL}$ of urine samples were added to the test tubes. The tubes were thoroughly mixed, and the initial absorbance was read spectrophotometrically against $340 \mathrm{~nm}$. Antihuman albumin antibody $(0.1 \mathrm{~mL})$ was added; the tubes were thoroughly mixed and incubated for 30 minutes. The final absorbance of each solution was read spectrophotometrically at $340 \mathrm{~nm}$, and the concentrations were calculated from the constructed standard curve.

2.6. Serum Creatinine and Urea Measurement. Serum creatinine and urea were assayed according to Jaffe Kinetic and Urease-GLDH Kinetic methods following protocol of commercial kit (LABKIT, Spain), respectively. 


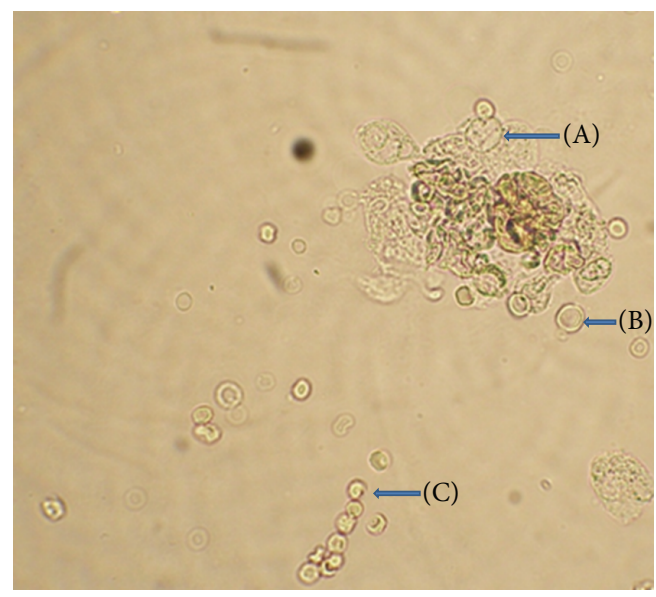

FIGURE 1: Microscopy of urinary sediments showing (A) epithelial cells, (B) RBCs, and (C) WBCs under high power objective $(\times 40 \mathrm{x})$.

Ethics approval was obtained from ethics committee of the institute and was in accordance with the 1975 Helsinki Declaration on Human Rights, as revised in Edinburgh 2000.

\section{Results}

All of the study patients revealed within normal range serum urea and creatinine levels at the time of admission. The routine urinalysis showed albuminuria in 10\% (4/40) and increased pus cells (WBCs) in 20\% (8/40) of VL cases before treatment with AmB (Figure 1). Hematuria was observed in $10 \%$ (4/40) patients. However, $15 / 40$ (37.5\%) of the study pediatric VL patients had detectable MA before treatment. The estimated GFR (eGFR) ranged between 60 and $89 \mathrm{~mL} / \mathrm{min} / 1.73 \mathrm{~m}^{2}$ in $27.5 \%(11 / 40)$ pediatric VL patients before treatment. The normal reference range of eGFR in paediatric group ranged between 92.3 and $122.5 \mathrm{~mL} / \mathrm{min} / 1.73 \mathrm{~m}^{2}$.

\section{Discussion}

Amphotericin B nephrotoxicity in children is a known complication. We investigated renal impairment in children suffering from VL before onset of Amphotericin B. In Sudanese VL patients, the renal injuries are mostly of glomerular in nature. The presence of this renal abnormality could be attributed to infiltration by infected macrophages and/or deposition of immune complexes in the glomeruli. The present study showed that urinary albumin excretion could be predictive for the development of de novo renal function impairment in pediatric VL cases.

The presence of significant microalbuminuria in $37.5 \%$ of VL patients with normal serum urea and creatinine was indicative of renal glomerular damage. However, in our present study, normal serum urea and creatinine levels probably point to the crudeness of these tests as indicators of renal damage. Another cross-sectional study of Lima Verde et al. on $50 \mathrm{VL}$ patients has reported decreased GFR in $28 \%$ of the cases, and our study reported decreased GFR in $27.5 \%$ of pediatric VL patients which indicates evidence of renal damage [12]. These abnormalities in glomerular filtration, urinary concentration, and acidification might be consistently associated with the chronic form of VL. Further, decreased GFR could be related to fluid loss, hypotension, and immunological glomerular damage. Since in this part of India the treatment is mostly being done by $\mathrm{AmB}$ in disease-endemic districts and drug combination therapy for $\mathrm{VL}$ is the trend worldwide, more sensitive investigations like eGFR, MA, and thorough microscopic examinations are needed to differentiate from early renal damage before start of chemotherapy.

In conclusion, glomerular involvement is the main renal injury in pediatric VL patients. The eGFR, MA, and microscopic examination maybe helpful in prediction of early renal damage. These parameters can provide an understanding of kidney injury and help treating doctors in decision making for safe and suitable antileishmanial treatment in pediatric group of patients.

\section{Conflict of Interests}

The authors declare that they have no conflict of interests.

\section{Authors' Contribution}

Neena Verma and Chandra Shekhar Lal contributed equally and treated as first author.

\section{Acknowledgments}

The authors wish to thank Mrs. Rakhi Kumari and Mr. Naresh Kumar Sinha, Technical Assistants, and Mr. Sudarshan Prasad, Lab Technician, for their valuable contribution in laboratory works.

\section{References}

[1] S. K. Bhattacharya, D. Sur, and J. Karbwang, "Childhood visceral leishmaniasis," Indian Journal of Medical Research, vol. 123, no. 3, pp. 353-356, 2006.

[2] A. M. El Hassan and E. A. G. Khalil, "Post-kala-azar dermal leishmaniasis: does it play a role in the transmission of Leishmania donovani in the Sudan?" Tropical Medicine and International Health, vol. 6, no. 9, pp. 743-744, 2001.

[3] E. E. Zijlstra and A. M. el-Hassan, "Leishmaniasis in Sudan. Visceral leishmaniasis," Transactions of the Royal Society of Tropical Medicine and Hygiene, vol. 95, supplement 1, pp. S27S58, 2001.

[4] E. A. G. Khalil, E. E. Zijlstra, P. A. Kager, and A. M. El Hassan, "Epidemiology and clinical manifestations of Leishmania donovani infection in two villages in an endemic area in eastern Sudan," Tropical Medicine and International Health, vol. 7, no. 1, pp. 35-44, 2002.

[5] A. Sartori, A. Viana de Oliveira, and M. C. Roque-Barreira, "Immune complex glomerulonephritis in experimental kalaazar," Parasite Immunology, vol. 9, no. 1, pp. 93-103, 1987.

[6] T. de Brito, S. Hoshino Shimizu, and V. Amato Neto, "Glomerular involvement in human kala azar. A light, immunofluorescent, and electron microscopic study based on kidney biopsies," 
American Journal of Tropical Medicine and Hygiene, vol. 24, no. 1, pp. 9-18, 1975.

[7] A. Poli, F. Abramo, F. Mancianti, M. Nigro, S. Pieri, and A. Bionda, "Renal involvement in canine leishmaniasis. A lightmicroscopic, immunohistochemical and electron-microscopic study," Nephron, vol. 57, no. 4, pp. 444-452, 1991.

[8] J. R. Weisinger, A. Pinto, and G. A. Velazquez, "Clinical and histological kidney involvement in human Kala-azar," American Journal of Tropical Medicine and Hygiene, vol. 27, no. 2, pp. 357359, 1978.

[9] F. A. Costa, J. L. Guerra, S. M. M. S. Silva, R. P. Klein, I. L. Mendonça, and H. Goto, "CD4 ${ }^{+} \mathrm{T}$ cells participate in the nephropathy of canine visceral leishmaniasis," Brazilian Journal of Medical and Biological Research, vol. 33, no. 12, pp. 1455-1458, 2000.

[10] M. Dutra, R. Martinelli, and E. M. de Carvalho, "Renal involvement in visceral leishmaniasis," American Journal of Kidney Diseases, vol. 6, no. 1, pp. 22-27, 1985.

[11] N. Salgado Filho, T. M. Ferreira, and J. M. Costa, "Involvement of the renal function in patients with visceral leishmaniasis (kala-azar)," Revista da Sociedade Brasileira de Medicina Tropical, vol. 36, no. 2, pp. 217-221, 2003.

[12] F. A. Lima Verde, F. A. Lima Verde, I. A. Lima Verde, G. B. Silva Jr., E. F. Daher, and E. M. Lima Verde, "Evaluation of renal function in human visceral leishmaniasis (kala-azar): a prospective study on 50 patients from Brazil," Journal of Nephrology, vol. 20, no. 4, pp. 430-436, 2007.

[13] F. A. A. Lima Verde, F. A. Lima Verde, E. de Francesco Daher, G. M. dos Santo, A. S. Neto, and E. M. Lima Verde, "Renal tubular dysfunction in human visceral leishmaniasis (Kalaazar)," Clinical Nephrology, vol. 71, no. 5, pp. 492-500, 2009.

[14] M. L. F. van Velthuysen and S. Florquin, "Glomerulopathy associated with parasitic infections," Clinical Microbiology Reviews, vol. 13, no. 1, pp. 55-66, 2000.

[15] V. Chaigne, Y. Knefati, R. Lafarge et al., "Acute renal failure complicating a visceral leishmaniasis," Nephrologie, vol. 25, no. 5, pp. 179-183, 2004.

[16] G. Efstratiadis, E. Boura, P. Giamalis et al., "Renal involvement in a patient with visceral leishmaniasis," Nephrology Dialysis Transplantation, vol. 21, no. 1, pp. 235-236, 2006.

[17] A. B. Libório, N. A. Rocha, M. J. C. Oliveira et al., "Acute kidney injury in children with visceral leishmaniasis," Pediatric Infectious Disease Journal, vol. 31, no. 5, pp. 451-454, 2012.

[18] M. Navarro, J. Bonet, J. Bonal, and R. Romero, "Secondary amyloidosis with irreversible acute renal failure caused by visceral leishmaniasis in a patient with aids," Nefrologia, vol. 26, no. 6, pp. 745-746, 2006.

[19] D. E. Varlam, M. M. Siddiq, L. A. Parton, and H. Rüssmann, "Apoptosis contributes to amphotericin B-induced nephrotoxicity," Antimicrobial Agents and Chemotherapy, vol. 45, no. 3, pp. 679-685, 2001.

[20] S. Sundar, H. Mehta, A. V. Suresh, S. P. Singh, M. Rai, and H. W. Murray, "Amphotericin B treatment for Indian visceral leishmaniasis: conventional versus lipid formulations," Clinical Infectious Diseases, vol. 38, no. 3, pp. 377-383, 2004.

[21] S. Sundar, T. K. Jha, C. P. Thakur, M. Mishra, V. P. Singh, and R. Buffels, "Single-dose liposomal amphotericin B in the treatment of visceral leishmaniasis in India: a multicenter study," Clinical Infectious Diseases, vol. 37, no. 6, pp. 800-804, 2003.

[22] S. Sundar, T. K. Jha, C. P. Thakur, M. Mishra, V. P. Singh, and R. Buffels, "Low-dose liposomal amphotericin B in refractory
Indian visceral leishmaniasis: a multicenter study," American Journal of Tropical Medicine and Hygiene, vol. 66, no. 2, pp. 143156, 2002.

[23] S. Sundar, G. Agrawal, M. Rai, M. K. Makharia, and H. W. Murray, "Treatment of Indian visceral leishmaniasis with single or daily infusions of low dose liposomal amphotericin B: randomised trial," British Medical Journal, vol. 323, no. 7310, pp. 419-422, 2001.

[24] C. S. Lal, A. Kumar, S. Kumar et al., "Hypocholesterolemia and increased triglyceride in pediatric visceral leishmaniasis," Clinica Chimica Acta, vol. 382, no. 1-2, pp. 151-153, 2007. 

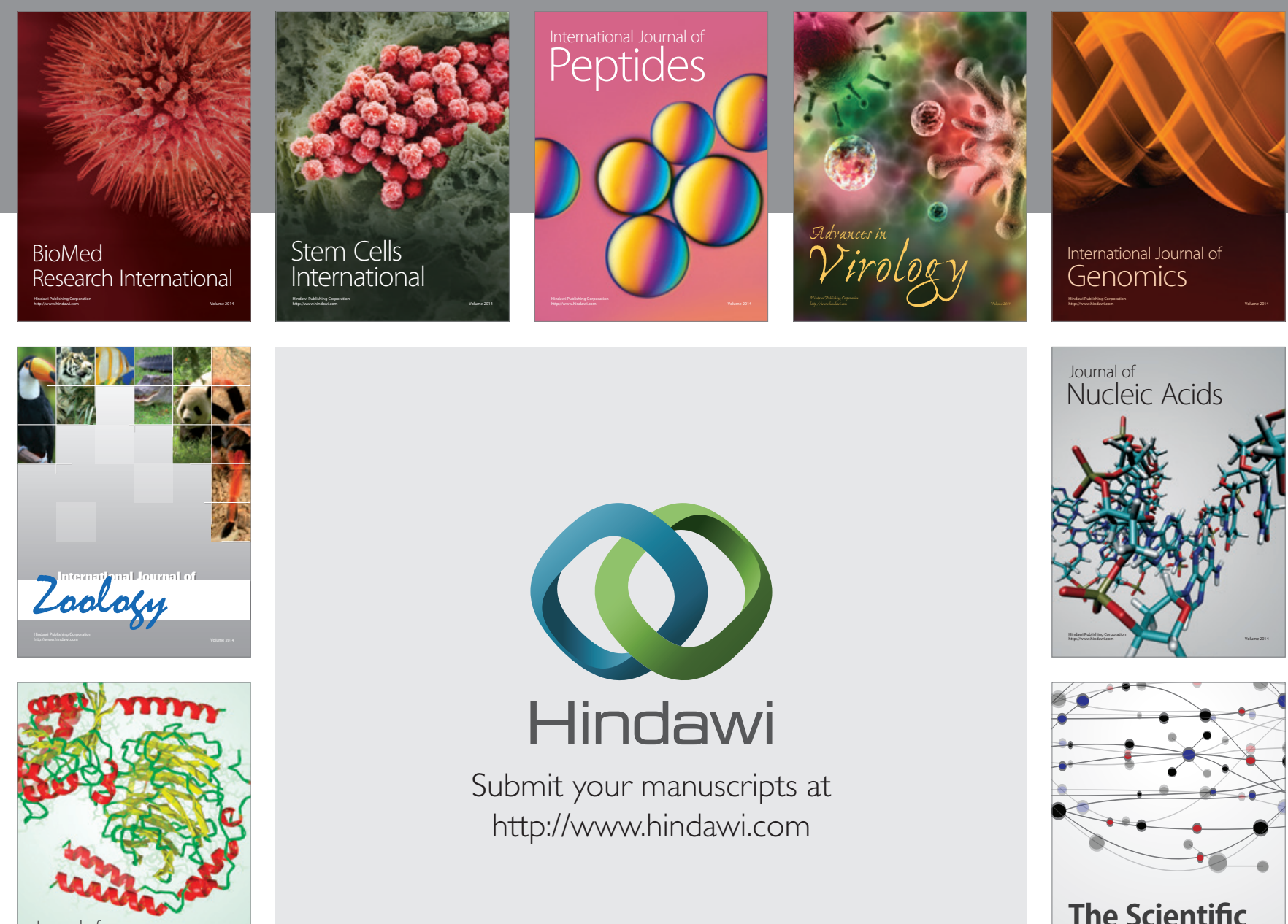

Submit your manuscripts at

http://www.hindawi.com

Journal of
Signal Transduction
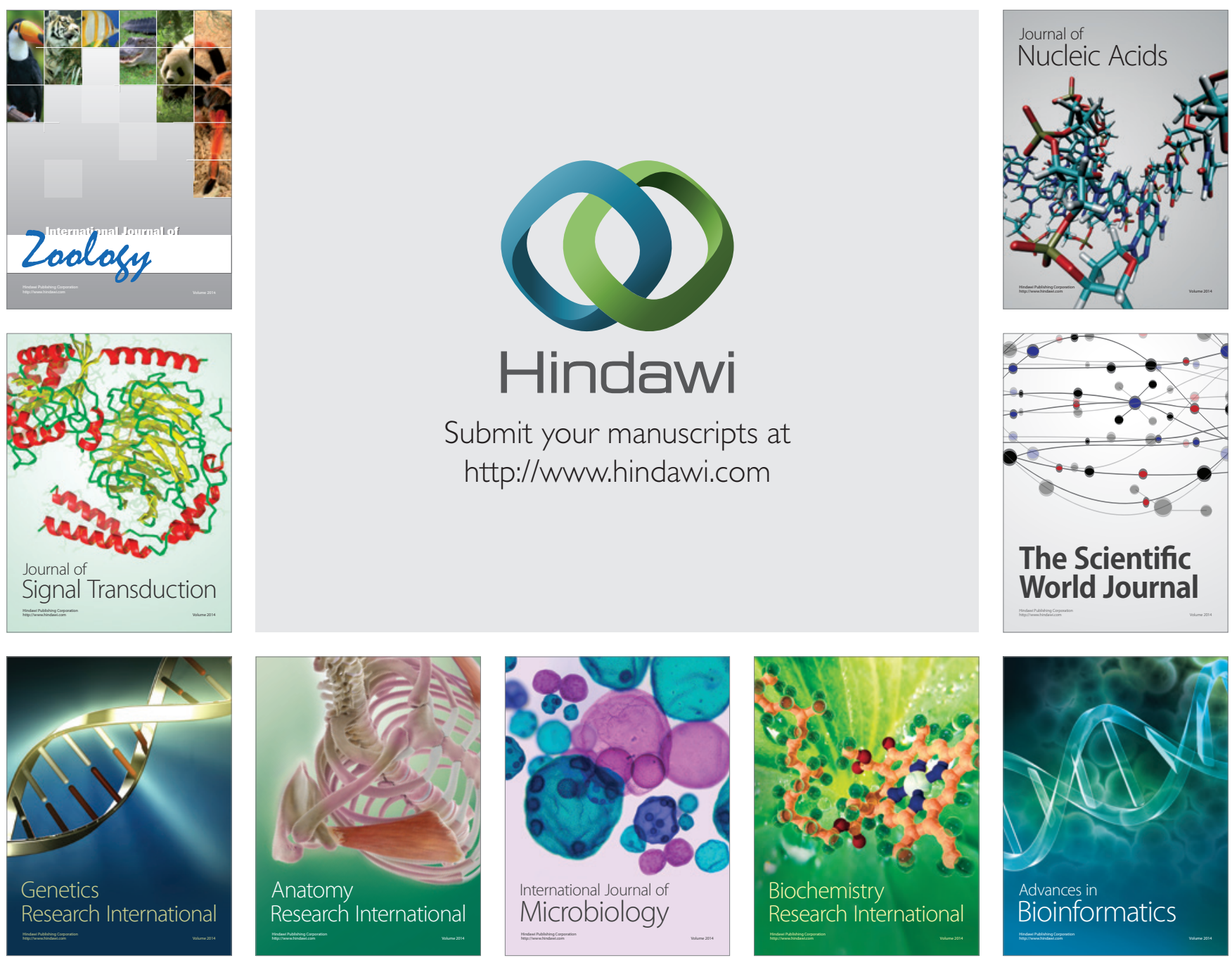

The Scientific World Journal
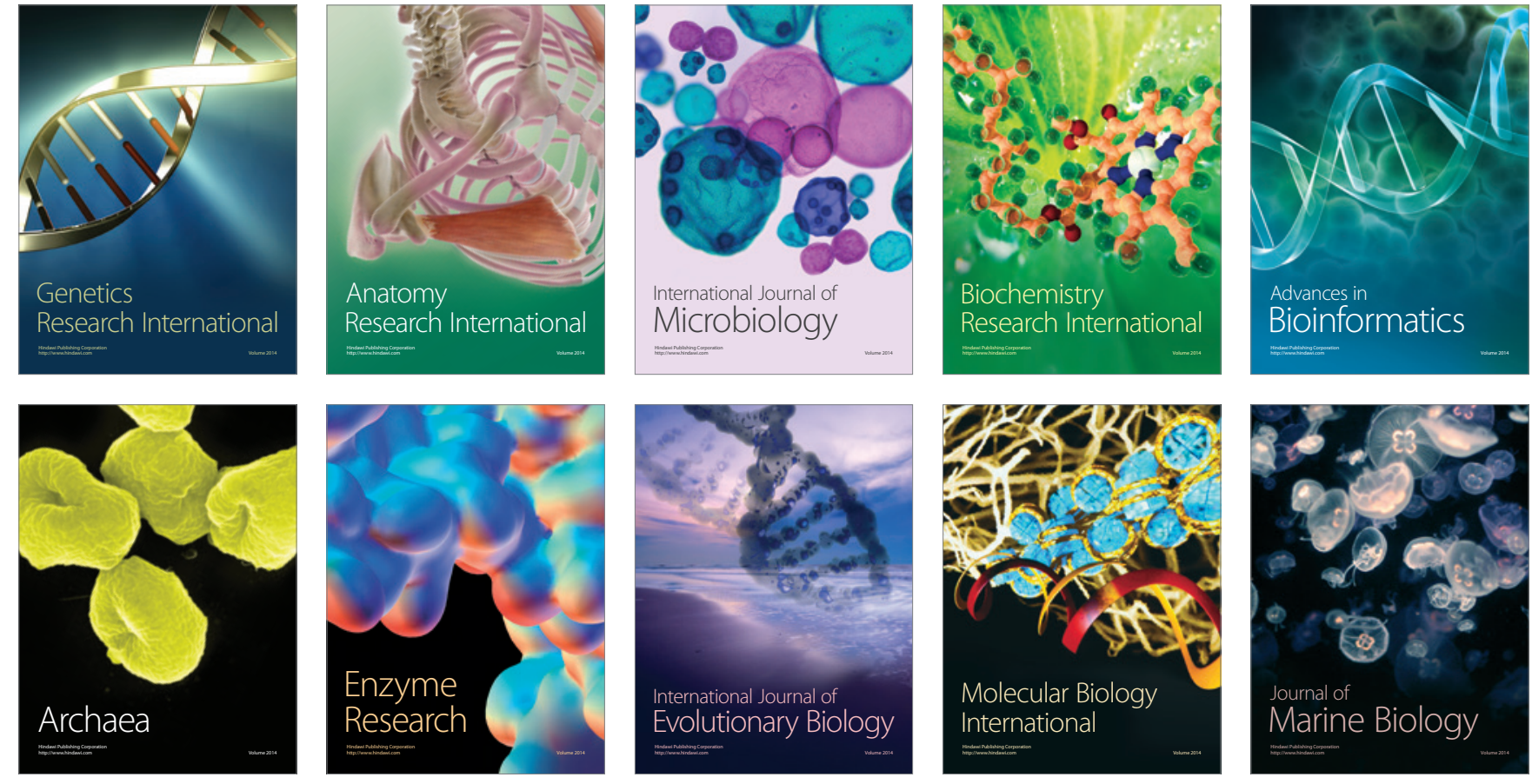